\title{
L'influence du livre des Proverbes sur le livre d'Osée,en relation avec les livres de Jérémie et d'Isaïe
}

\author{
BERNARd GOSSE (ANTONY, FranCE)
}

\begin{abstract}
The Book of Proverbs plays an important role in the redaction of the Book of Hosea in connection with the conclusion of Hosea in 14, 10. This influence is particularly related to the transfer upon the Northern Kingdom of some condemnations pronounced about the Southern Kingdom in the Books of Isaiah and Jeremiah.

RÉSUMÉ

Le livre des Proverbes joue un rôle important dans la rédaction du livre d'Osée en relation à la conclusion de 14,10. Cette influence est particulièrement liée au transfert sur le royaume du Nord de plusieurs condamnations concernant le royaume du Sud dans les livres d'Isaïe et de Jérémie.
\end{abstract}

Key concepts : Proverbs, Hosea, Jeremiah, Northern kingdom, Southern kingdom.

\section{A INTRODUCTION}

Dans mon livre, L'Influence du livre des Proverbes sur les rédactions biblique à l'époque perse, j'ai souligné le rôle du livre des Proverbes, dans la rédaction du Psautier et d'autres livres Bibliques, particulièrement les trois grands livres prophétiques. ${ }^{1}$ Dans cet article nous allons examiner le rôle du livre des Proverbes, dans le livre d'Osée, en lien avec les livres de Jérémie et d'Isaïe.

* L'article présenté : 17/12/2014 ; accepté : 29/01/2015. À citer : Bernard Gosse, «L'influence du livre des Proverbes sur le livre d'Osée, en relation avec les livres de Jérémie et d'Isaïe, » OTE 28/1(2015) : 113-120, DOI : http:// dx.doi.org/10.17159/2312-3621/2015/v28n1a8

1 Bernard Gosse, L'influence du livre des Proverbes sur les rédactions bibliques à l'époque Perse (Supplément n 14 à Transeuphratène; Paris: Gabalda, 2008). Pour les livres, d'Isaïe 97-144; Jérémie 145-186; Ezéchiel 187-210. Pour les livres des Rois voir encore Bernard Gosse, «L'artisanat de Tyr comme modèle de la sagesse Yahviste de Salomon», in Phéniciens d'Orient et d'Occident: Mélanges Josette Elayi (éd. André Lemaire, Bertrand Dufour et Fabian Pfitzmann; CIPOA II; Paris; Jean Maisonnneuve, 2014), 43-50. Pour le Psautier, voir Phil J. Botha, «Pride and the Suffering of the Poor in the Persian Period: Psalm 12 in its Post-Exilic Context», OTE 25 (2012): 40-56; Phil J. Botha, «Psalm 91 and its Wisdom Connection», OTE 25 


\section{B OSÉE 14,10, L'INVITATION AU LECTEUR, Á PRENDRE UNE ATTITUDE OPOSÉE, À LA FOLIE DES ISRAÉLITES DU ROYAUME DU NORD}

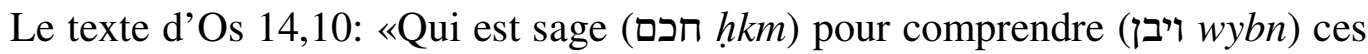

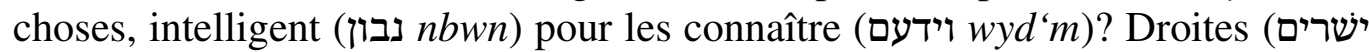
yšrym) sont les voies (דרכי drky) de Yahvé, les justes (וצדקים wșdqym) y

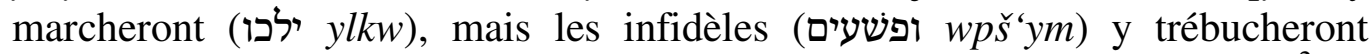
(יכשלו $y k$ Šslw)», apparaît comme une conclusion de sagesse au livre d'Osée. ${ }^{2}$ Le lecteur est invité à avoir un comportement, différent de celui des habitants du royaume du Nord, selon Os 4,14: «je ne châtierai pas vos filles pour leurs prostitutions, ni vos brus pour leurs adultères; car eux-mêmes vont à l'écart avec les prostituées, ils sacrifient avec les hiérodules, et le peuple (יעם b' $w^{\prime} m$ ), sans discernement (לאיבין l'ybyn), va à sa perte (ליבט) ylbt)!» Ces passages correspondent, aux deux seuls emplois du verbe ביא byn dans le livre d'Osée. Ce point souligne que le lecteur, est lui invité, à faire preuve de discernement. Or Os 4,14 manifeste encore autrement, des liens privilégiés avec le livre des Proverbes. Ainsi le verbe לבט lbt apparait en Os 4,14: Prov 10,8.10 pour toute

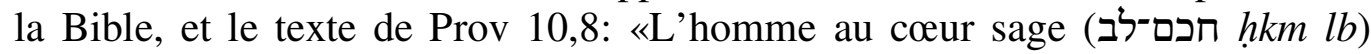

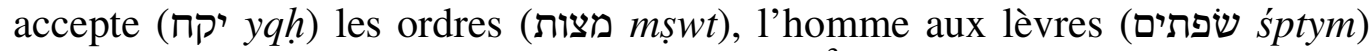
folles (ואויל w'wyl) court à sa perte (ילבט ylbt)», ${ }^{3}$ correspond bien à l'opposition entre l'attitude supposée, du lecteur du livre d'Osée et les comportements attribués aux Israélites du royaume du Nord. La fin d'Os 4,14 apparaît comme un «dit» de sagesse, ce qui correspond également, à l'usage du verbe au singulier, en dépendance du sujet עם ' $m$ sans article. ${ }^{4}$ Le livre d'Osée témoigne

(2012): 260-276; Phil J. Botha, «Poetry and Perlocution in Psalm 26», OTE 24 (2011): 30-48; Phil J. Botha, "'I am like a Green Olive Tree': The Wisdom Context of Psalm 52», HTS 69/1 (2013); Art. \#1962, 8 pages; DOI: 10.4102/hts .v69i1.1962; Phil J. Botha, «Interpreting 'Torah' in Psalm 1 in the Light of Psalm 119», HTS 68/1 (2012); Art. \#1274, 7 pages; DOI: 10.4102/hts.v68i1.1274; Annette Potgieter, «Walking Wisely: Sapiential Influence in Psalm 26», HTS 69/1 (2013); Art. \#1378; 5 pages; DOI: 10.4102/hts.v69i1.1378.

Andrew A. Macintosh, Hosea (Edinburgh: T \& T Clark, 1997), 582: «The verse, replete with the language of the Wisdom literature (see below), is added to the collected prophecies of Hosea ('these matters') and suggests to the reader the lesson that he should draw from them».

3 Francis I. Andersen and David N. Freedman, Hosea (Garden City, N.Y.: Doubleday \& Company, 1980), 371: «Ruin is the fate of the foolish talker in the two other places where the verb ילבט ylbt occurs (Prov 10:8,10)».

4 Andersen et Freedman, Hosea, 371: «This proverb does not make the meaning of the verb any clearer, but it suggests that this live, which is rhythmical and alliterative, is part of a Wisdom saying; this might account for the singular number of the verb, as "people", usually without the article, always has a singular verb in Proverbs. Note especially עם לאיעז 'am lo'-'az (Prov 30:25) and 'așum (Prov 30:26)». 
du reste, d'une autre attitude dénoncée, en Prov 10,8 et attribuée aux israélites du Nord en Os 9,7 : "Le prophète est fou (אויל הנביא 'wyl hnby'), , l'inspiré délire (משגע איש הרוח 'mšg ' 'yš hrwh)», avec אויל 'wyl: Os 9,7; Jr 4,22; Is 19,11; 35,8; Ps 107,17; Jb 5,2.3; et 18 emplois dans le livre des Proverbes, dont Prov 10,8.10.14.21, pour toute la Bible. L'attitude à laquelle est invité le lecteur, s'oppose encore, à celle attribuée aux israélites du Nord en Os 13,13: «Les douleurs de l'enfantement surviennent pour lui, mais c'est un enfant stupide (לא חכם l' hkm) ${ }^{6}$ : il est à terme et ne quitte pas le sein maternel!», avec les deux seuls emplois, de חכם לחם hans le livre d'Osée en 13,13 et 14,10, alors que le substantif, est bien entendu courant dans le livre des Proverbes, ainsi que le verbe. Le terme פשעים pšym d'Osée 14,10 renvoie aux

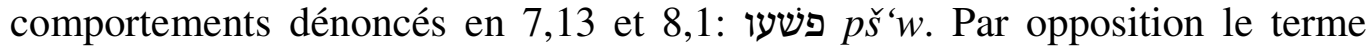
צדיק șdyq: Os 14,10, seule attestation du livre d'Osée, est très fréquent dans les Proverbes.

\section{OSÉE 4,1-6.10 SELON LE LIVRE DES PROVERBES ET CELUI DE JÉRÉMIE}

Le texte d'Os 4,1: «Ecoutez la parole de Yahvé, enfants d'Israël, car Yahvé est en procès avec habitants du pays: il n'y a ni fidélité (אמת (אד) 'mt) ni piété $h s d$ ), ni connaissance (דעת $\left.d^{\prime} t\right)^{7}$ de Dieu ${ }^{8}$ dans le pays», ${ }^{9}$ situe le comportement des enfants d'Israël en opposition à Prov 3,3: «Que piété (חסד hsd) et fidélité (ואמת w'mt) ne te quittent! Fixe-les à ton cou, inscris-les sur la tablette de ton cœur» et Prov 2,10: «Quand la sagesse (חכמה hlkmh) entrera dans ton cœur que le savoir (ודעת wd't) fera les délices de ton âme».

Si la rédaction du livre d'Osée, témoigne de l'influence du livre des Proverbes, cela le rapproche du cas de nombreux livres bibliques, qui ont subi cette influence à l'époque Perse, y compris le livre de Jérémie. ${ }^{10}$ Or J: M: Bos,

5 J. Andrew Dearman, The Book of Hosea (Grand Rapids: William B. Eerdmans Publishing Company, 2010), 244: «The foolish prophet, according to 9:7, is one who does not recognize the presence of the time of judgment».

6 Dearman, Hosea, 327: «Among other things, wisdom is concerned with recognizing the right time for an action».

7 Douglas Stuart, Hosea-Jonah (Nashville: Thomas Nelson Publishers, 1987), 75: «The use of דעת אלהים d't 'lhym elsewhere in Hosea (6:6), along with other expressions of knowing God based upon the rost ידע $y d^{\prime}$ ' (2:22(20); 4:6; 5:4; 8:2; 13:4; etc) demonstrates how in Hosea the term represents the essence of the covenant relationship between God and his people». Voir Pr 2,17.

8 Macintosh, Hosea, 128: «'Knowledge of God'...It is akin to the fear of Yahweh (cf. Prov 2.5)...».

9 אמת 'mt: Os 4,1; Prov 3,3; 8,7; 11,18; 12,19; 14,22.25; 16,6; 20,28; 22,21; 23,23; 29,14 חלד hsd: Os 2,21; 4,1; 6,4.6;10,12; 12,7; Prov 3,3;11,17; 14,22.34; 16,6; 19,22; 20,6.28; 21,21; 31,26 דעת d't: Os 4,1.6; 6,6; Prov 1,4.7.22.29; 2,5.6.10; 3,20; 5,$2 ; 8,9.10 .12 \ldots$

10 Gosse, L'influence du livre, 145-185. 
a proposé d'attribuer le livre d'Osée, à l'élite non-royale de Judée à l'époque post monarchique. ${ }^{11}$ Il note de plus, plusieurs liens avec les livres prophétiques, dont le livre de Jérémie. ${ }^{12}$

Dans cette perspective, on peut noter qu'Os 4,2: «mais parjure et

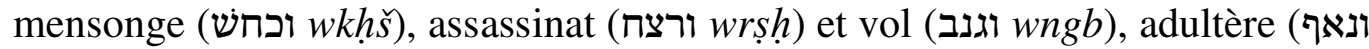
$w n ' p$ ) et violence, et le sang versé succède au sang versé», recoupe la description pour le royaume du Sud, selon Jr 7,9 tuer (רצח ונאף (ומאף $w h s ̌ b$ ' lšqr), encenser Baal, suivre les dieux étrangers que vous ne connaissez pas». Au niveau du vocabulaire on peut souligner: רצִ $r$ (infinitif kal): Os 4,2; Jr 7,9, seuls emplois bibliques et de même pour גנב gnb (infinitif kal): Os 4,2; Jr 7,9; Ex 22,11; נאף n'p (infinitif kal): Os 4,2; Jr 7,9; 23,14. On peut y ajouter Is 59,13: «nous révolter, renier (וכחש wkȟs) Yahvé, cesser de suivre notre Dieu; proférer violence et révolte, concevoir et méditer le mensonge», avec כחש kȟs (infinitif pi 'el): Os 4,2; Is 59,13; Za 13,4, pour toute la Bible.

De même le texte d'Os 4,3: «Voilà pourquoi le pays est en deuil ( על־כן ת 'l kn t'bl h'rṣ) et tous ses habitants dépérissent (ואמלל הארץ w'mll), jusqu'aux bêtes des champs et aux oiseaux du ciel, et même les poissons de la mer disparaîtront», transpose sur le royaume du Nord, ce qui est affirmé au sujet de Juda, en Jr 14,2: «Juda est dans le deuil (אבלה 'blh) et ses villes dépérissent (אמללו'mllw): elles s'abîmes vers la terre, le cri de Jérusalem s'élève», avec: אבל 'bl (kal): Os 4,3; 10,5: Jr 4,28 ${ }^{14} ; 12,4.11 ; 14,2 ; 23,10$, et אמל 'ml: Os 4,3; Jr 14,2; 15,9, pour ces deux livres.

Dans le cas d'Os 4,4-5: «4 Pourtant que nul n'intente procès (ירב yrb), que nul ne réprimande (יוכח ywkh)! C'est avec toi, prêtre (כהן khn), que je suis en procès (כמריבי וכשרלת (כmryby). 5 Tu trébucheras en plein jour, le

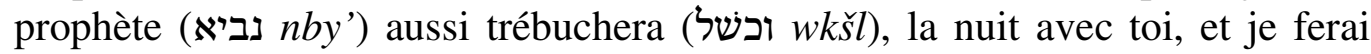
périr ta mère», on relève le vocabulaire des Proverbes avec: יכח $y k h$ : Os 4,4; Prov 3,$12 ; 9,7.8 .8 ; 15,12 ; 19,25 ; 24,25 ; 25,12 ; 28,23 ; 30,6$; ריב ryb: Os 2,4; 4,4.4; 5,13; 10,6; Prov 3,30; 22,23; 23,11; 25,8.9; כשל kšl: Os 4,5; 5,5.5; 14,2.10; Prov 4,12.16.16.19; 24,16.17. Mais ce passage du livre d'Osée,

11 James M. Bos, Reconsidering the Date and Provenance of the Book of Hosea: The Case for Persian-Period Yehud (New York: T \& T Clark, 2013).

12 Bos, Reconsidering, 182 (index).

13 Dearman, Hosea, 151: «The same three transgressions occur in an accusation in Jer. 7:9, employing the infinitive absolute to characterize the acts, as in Hos. 4:2, but with yet a different sequence».

14 Jr 4,28: על־זאת תאבל הארץ z't t'bl h'rṣ. Andersen et Freedman, Hosea, 340-341 (Os 4,3b): «It resembles Jer 4:23-28, which describes the ruination of all nature in the last judgments of God. In Jr 4:28, "the land is dried up"...». 
introduit également le vocabulaire du prêtre ${ }^{15}$ et du prophète, absent du livre des Proverbes, conformément à Jr 2,8-9: «8 Les prêtres (הכהנים hkhnym) n'ont pas dit: "Où est Yahvé?" Les dépositaires de la Loi ne m’ont pas connu; les pasteurs se sont révoltés contre moi; les prophètes (והנביאים whnby'ym) ont prophétisé par Baal, ils ont suivi des Impuissants. 9 Aussi je suis en procès (אריב'ryb) contre vous - oracle de Yahvé - et je suis en procès (אריב'ryb) contre les fils de vos fils». L'influence du livre de Jérémie est d'autant plus probable que le prophète cultuel était inconnu dans le Nord. ${ }^{16}$

On relève encore le vocabulaire du livre des Proverbes en Os 4,6: «Mon peuple périt, faute de connaissance (הדעת $h d^{\prime} t$ ). Puisque toi, tu as rejeté la connaissance (הדעת $h d^{\prime} t$ ), je te rejetterai de mon sacerdoce; puisque tu as oublié (ותשכח ותtškh) l'enseignement (תורת twrt) de ton Dieu, à mon tour, j'oublierai tes fils», ${ }^{17}$ avec: דעת $d$ 't: Os 4,1.6; 6,6; Prov 1,4.7.22.29; 2,5.6.10; 3,20; 5,2; 8,9.10.12...; תורה twrh : Os 4,6; 8,1.12; Prov 1,8; 3,1; 4,2: 6,20.23; 7,2; 13,14; 28,4.7.9; 29,18; 31,26: שכח šl Os 2,15; 4,6; 8,14; 13,6; Prov 2,17; 3,$1 ; 4,5 ; 31,5.7$

Il en est de même, en Os 4,10: «Ils mangeront, mais sans se rassasier, ils se prostitueront, mais sans s'accroître, car ils ont abandonné (עזבו 'zbw) Yahvé pour se livrer 11 à la prostitution», avec: עזב 'zb: Os 4,10; Prov 2,13.17; 3,3; $4,2.6 ; 9,6 ; 10,17 ; 15,10 ; 27,10 ; 28,4.13$

\section{OSÉE 6,6 SELON LE LIVRE DES PROVERBES}

La doctrine d'Os 6,6: «Car c'est l'amour (חסד hsd) qui me plaît et non les sacrifices (זבח ודעת) ו ודת $z b h$ 't $t$ ) de la connaissance Dieu plutôt que les holocaustes», ${ }^{18}$ est conforme à Prov 21,3: «Pratiquer la justice et le droit vaut, pour Yahvé, mieux que le sacrifice (מזבח $m z b h) »$, avec de plus: דעת d't: Os

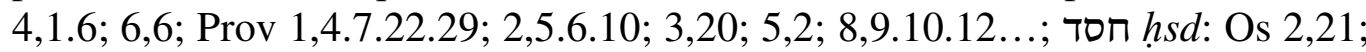

15 Macintosh, Hosea, 135, note d: כהן khn is understood as a vocative and as a collective singular. The absence of the article in vocatives which are collective singular is confirmed by Mic 6.8; Zech 11.2; Prov 24.15; Eccles 11.9»; 136: «The priests are addressed as a collective singular (note d). Attempts to interpret the passage (vv. 4-6) in terms of an historical altercation between a single particular priest (unknown to us) and Hosea are not convincing».

16 Stuart, Hosea-Jonah, 77: «and ignores the fact that at the Jerusalem temple prophets served the cult along with the priests (Isa 28:7; Jer 2:8; 4:9; 5:31; 6:13; 8:10; $14: 18 ; 18: 18 ; 23: 11$; Mic $3: 11)$. It is unlikely that the well-attested southern practice of priest-prophet cooperation was unknown in the north».

17 Dearman, Hosea, 158: «This is recognized in the succinct formulation of Jer 2.8...».

18 Dearman, Hosea, 196 (Os 6,6): «YHWH's poetic affirmation is also proverb-like in form, i.e., a wise saying that can be applied in a variety of circumstances. It is employed that way, as a saying apart from its Hosea context, in the Gospel of Matthew $(9: 13 ; 12: 7) »$. 
4,$1 ; 6,4.6 ; 10,12 ; 12,7 ;$ Prov 3,$3 ; 11,17 ; 14,22.34 ; 16,6 ; 19,22 ; 20,6.28 ; 21,21$; 31,26

\section{E OSÉE 2,1-2 SELON Jr 33,22}

Le texte d'Os 2,1-2: «1 Le nombre (מספר mspr) des enfants d'Israël sera comme le sable de la mer (כחול הים khwl hym), qu'on ne peut ni mesurer (מדר ymd) ni compter (יספר yspr); au lieu même où on leur disait: "Vous n'êtes pas mon peuple", on leur dira: "Fils du Dieu vivant". 2 Les enfants de Juda (בני־ ישריה יהודה bny yhwdh) et les enfants d'Israël (ובנייישראל wony ys'l) se réuniront

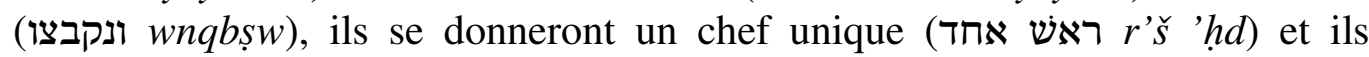
déborderont hors du pays, car il sera grand le jour de Yizréel», apparaît en relation avec la perspective de Jr 33,22 ${ }^{19}$ : «Comme l'armée des cieux qui ne peut être dénombrée (מישפר yspr), comme le sable de la mer (חול הים hwl hym) qui ne peut être compté (מימדymd), ainsi multiplierai-je la postérité de David mon serviteur, et les lévites qui assurent mon service», avec: חול מרול $h w l$ : Os 2,1; Jr 5,22; 15,8; 33,22; מדד mdd: Os 2,1; Jr 31,37; 33,22; ספר spr: Os 2,1 seul usage du livre d'Osée; Jr 33,22 et nombreux emplois dans le livre de Jérémie.

Le texte de Jr 33,22 est inclus dans un passage du livre de Jérémie, très proche des préoccupations des Chroniques, avec une réhabilitation de David en temps qu'instaurateur du personnel du temple. ${ }^{20}$ Or Os 2,1-2 présente également des perspectives conforme à Jr 32,30*.37*.39*: «30 Car les enfants d'Israël (בני-ישראל bny yśr'l) et ceux de Juda (ובני יהודה wbny yhwdh)... 37 Moi, je vais les rassembler (מקבצם mqbșm)... 39 je leur donnerai un seul cœur et une seule manière d'agir (לב אחד ודרך אח :lb 'hd wdrk 'hd)...», avec: קבץ qbș: Os 2,2; 8,10; 9,6; Jr 23,3; 29,14; 31,8.10;32,37; 40,15; 49,5.14, et ' $h d$ : Os 2,2 seul emploi du livre d'Osée; Jr 3,14; 10,8; 24,2.2; 32,39; 35,2; 51,$60 ; 52,1 \cdot 1.20 .21 .22 .25$. On peut penser que dans le cas de la perspective anti monarchique du livre d'Osée l'expression ראש אחד r's 'hd, ${ }^{21}$ peut faire allusion au grand prêtre, avec une expression à rapprocher de celle de Jr 52,24: khn $h r$ 'š. La position du livre d'Osée apparaît donc beaucoup plus ouverte que celle de $\mathrm{Jr} 33,22$ qui s'aligne sur la perspective davidico-cultuel des livres des Chroniques.

19 Stuart, Hosea-Jonah, 38: כחול הים"khwl hym... Jer 33:22, on the other hand, parallels very closely the eschatological emphasis of the term in Hosea».

20 Voir le rôle de David et des lévites en Jr 33,18.22.24.26, et en 1 Ch 16, la citation du Ps 105 se concluant par 105,15, les messies devant être compris comme les prêtres oints dans les Chroniques et les prophètes devant être interprétés comme les lévites dans les Chroniques.

21 Andersen et Freedman, Hosea, 200: «The main points made are the greatness of the coming day ( $\mathrm{v} 2 \mathrm{~b}$ ), clearly a day of exultation; the great increase in population ( $\mathrm{v}$ 1a); the reversal of one of the negative names (v 1b); and the reunion of the separated kingdoms under a single ruler (v 2a)». 


\section{F LES ENFANTS D'OSÉE ET D'ISAÏE, LE LIVRE D'OSÉE ET CELUI D'ISAÏE ${ }^{22}$}

Le texte d'Os 1,3: «Il alla ( וילך wylk) donc prendre (זיקחwyqh) Gomer, fille de

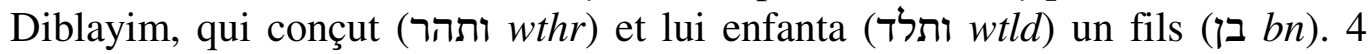
Yahvé lui dit: “Appelle-le (קרא qr') du nom (שמו šmw) de Yizréel, car encore un peu de temps (מעט $m$ 't $t$ ), et je châtierai la maison de Jéhu pour le sang versé à Yizréel, et je mettrai fin à la royauté de la maison d'Israël”.», au-delà même d'un vocabulaire commun, présente un scénario très proche de celui d'Is 8,3-4: «3 Puis je m'approchai de la prophétesse, elle conçut (ותהר wthr) et enfanta

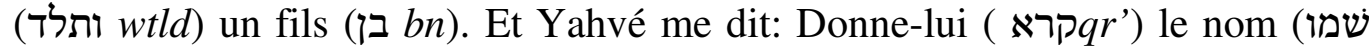
$\check{s} m w$ ) de Maher-Shalal Hash-Baz, 4 car avant que le garçon ne sache dire "papa" et "maman", on enlèvera la richesse de Damas et le butin de Samarie, en présence du roi d'Assur». ${ }^{23}$

On peut de plus remarquer que Os 1,1: «Parole de Yahvé qui fut adressée à Osée (הושע $\left.h w \check{s}^{\prime}\right),{ }^{24}$ fils de Beéri, au temps d'Ozias, de Yotam, d'Achaz et d'Ezéchias, rois de Juda, et au temps de Jéroboam, fils de Joas, roi d'Israël», ${ }^{25}$ correspond en une transposition sur le royaume du Nord d'Is 1,1: «Vision d'Isaïe (ישעיהו yš 'yhw), fils d'Amoç, qu'il reçut au sujet de Juda et de Jérusalem, au temps d'Ozias, de Yotam, d'Achaz et d'Ezéchias, rois de Juda». Le thème de la prostitution en Os 1,2, correspond pour le royaume du Sud à l'affirmation d'Is 1,21 pour Jérusalem. ${ }^{26} \mathrm{~L}$ 'annonce de la fin de la royauté dans le royaume du Nord, en Os 1,4, correspond également à l'enseignement du livre d'Isaïe, où David disparaît à la fin du livre.

\section{BIBLIOGRAPHIE}

Andersen, Francis I. et David N. Freedman. Hosea. Garden City, N.Y.: Doubleday \& Company, 1980.

Bos, James M. Reconsidering the Date and Provenance of the Book of Hosea: The Case for Persian-Period Yehud. New York: T \& T Clark, 2013.

Botha, Phil J. «Poetry and Perlocution in Psalm 26». Old Testament Essays 24 (2011): $30-48$.

22 Kay Weissflog, Zeichen und Sinnbilder: Die Kinder der Propheten Jesaja und Hosea (Leipzig: Evangelische Verlangsanstalt, 2011).

23 Voir Weissflog, Zeichen, 525-526.

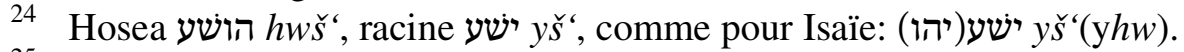

25 Stuart, Hosea-Jonah, 21: «The inclusion of Hezekiah (725-686: 715-686 as sole regent) in the southern list suggests that Hosea was, if only briefly (725-722?) a contemporary of Isaiah (cf. Isa 1:1) » (lecture historique, nous faisons une lecture littéraire). Weissflog, Zeichen, 259: «Hos 1,1 nennt dieselben judäischen Könige wie Jes 1,1. Beide Prophetenbücher rücken dadurch in eine deutliche Nähe zueinander».

26 Dans le libre d'Isaïe nous avons un retournement semblable à celui du livre d'Osée en Is 62,4, voir Weissflog, Zeichen, 278. 
120 Gosse, "L’influence du livre,” OTE 28/1 (2015): 113-120

. «Pride and the Suffering of the Poor in the Persian Period: Psalm 12 in its

Post-Exilic Context». Old Testament Essays 25 (2012): 40-56.

. «Psalm 91 and its Wisdom Connection». Old Testament Essays 25 (2012):

260-276

. «I am like a Green Olive Tree': The Wisdom Context of Psalm 52». HTS

Teologiese Studies / Theological Studies 69/1 (2013). Art. \#1962. 8 pages. DOI:

10.4102/hts .v69i1.1962.

. «Interpreting 'Torah' in Psalm 1 in the Light of Psalm 119». HTS

Teologiese Studies / Theological Studies 68/1 (2012). Art. \#1274. 7 pages. DOI: 10.4102/hts.v68i1.1274.

Potgieter, Annette. «Walking Wisely: Sapiential Influence in Psalm 26». HTS

Teologiese Studies / Theological Studies 69/1 (2013). Art. \#1378. 5 pages. DOI: 10.4102/hts.v69i1.1378.

Dearman, J. Andrew. The Book of Hosea. Grand Rapids: William B. Eerdmans Publishing Company, 2010.

Gosse, Bernard. L'influence du livre des proverbes sur les rédactions bibliques à l'époque Perse. Supplément n 14 à Transeuphratène. Paris: Gabalda, 2008. . «L'artisanat de Tyr comme modèle de la sagesse Yahviste de Salomon». Pages 43-50 dans Phéniciens d'Orient et d'Occident: Mélanges Elayi. Edité par André Lemaire, Bertrand Dufour et Fabian Pfitzmann. Cahiers de l'institut du proche-orient ancien du Collège de France 2. Paris: Jean Maisonnneuve, 2014.

Macintosh, Andrew A. Hosea. Edinburgh: T \& T Clark, 1997.

Stuart, Douglas. Hosea-Jonah. Nashville: Thomas Nelson Publishers, 1987.

Weissflog, Kay. Zeichen und Sinnbider: Die Kinder der Propheten Jesaja und Hosea. Leipzig: Evangelische Verlangsanstalt, 2011.

Bernard Gosse, 4 Résidence Opéra, 92160 Antony, France. E-mail : gosse.bernard@wanadoo.fr. 